$B N L--51455$

DE82 014261

\title{
A MULTI-LAYER UNIVERSAL CORRECTION MAGNET
}

\author{
G. Parzen
}

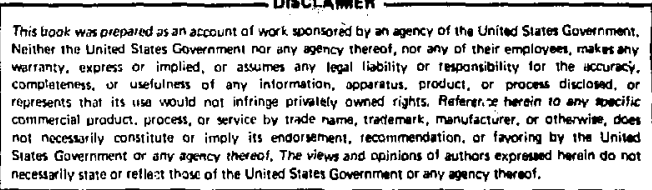

August 1981

\section{ACCELERATOR DEPABTMENT}

\section{BROOKHAVEN NATIONAL LABORATORY \\ UPTON, NEW YORK 11973}


ABSTRACT

This paper presents an approach for constructing a universal correction magnet in which the return currents play an active role in determining the field. The return currents are not hidden by the iron shield. The coil is wound in many layers, instead of just one layer. Each layer has a particular symetry, and generates a particular class of field multipoles such that the location of the return current for each independently excited current block is clear. Three layers may be sufficient in many cases. This approach is applied to the ISABELLE storage accelerator correction system. 


\section{INTRODUCTION}

This paper presents an approach for constructing a universal correction magnet in which the return currents play an active role in determining the field. This approach is applied to the ISABELLE correction system.

A universal correction magnet is a magnet in which the correction field is generated by an arrangement of current blocks, which are all independently excited. Thus by proper excitation of each current block, one can generate an arbitrary correction field within certain limits, which are primarily determined by the number of current blocks. Usually the return current for each current block is hidden by the iron shield and plays no direct role in shaping the field correction. In the approach being presented here, the return currents are not hidden by the iron shield. In this approach, the coil is wound in many layers instead of just one layer. Each layer has a particular symmetry, and generates a particular class of field multipoles such that the location of the return current for each current block is clear. These layers are defined as follows.

Layer 1. This layer generates the field multipoles $b_{k-1}$ where $k=2 n+1, n=0,1,2,3 \ldots$ Thus $k=1,3,5,7, \ldots$, and this 1 ayer generates all the even multipoles $b_{0}, b_{2}, b_{4}, b_{6}, \ldots$ The multipole field is given by $B_{o} b_{m} x^{m}$, where $B_{o}$ is some relevant standard field. For this layer, the return current for the current block at the angle $\theta$ with the median plane is at $\pi-\theta$. The arrangement of the current blocks in layer 1 is shown in the middle layer of the magnet shown in Fig. 1.

Layer 2. This layer generates the field multipoles $b_{k-1}$, where $\mathrm{k}=(2 \mathrm{n}+1) \times 2, \mathrm{n}=0,1,2,3, \ldots$. Then $\mathrm{k}=2,6,10,14 \ldots$ and the 1 ayer generates the odd multipoles $b_{1}, b_{5}, b_{9}, b_{13} \ldots$ For the current blocks in the range $0<\theta<\pi / 2$, the return current for the current block at $\theta$ is at $\pi / 2-\theta$. This pattern repeats itself as shown in the inner layer of the magnet shown in Fig. 1.

Layer 3. This layer generates the multipoles $b_{3}, b_{11}, b_{19} \ldots$, or $b_{k-1}$ where $k=(2 n+1) \times 4, n=0,1,2, \ldots$ In the interval $\theta=0$ to $\pi / 4$, the return current for the current block at $\theta$ is at $\pi / 4-\theta$. This pattern repeats itself as shown in the outer layer of the magnet shown in Fig. 1 .

Further layers are described in Table 1. In principle, an infinite number of layers are required to generate all field multipoles. In practice, it seems likely that 3 layers may be sufficient. Figure 1 shows a 3 layer design which can generate all the multipoles up to and including $b_{13}$ with the exception of $b_{7}$. The absence of the $b_{7}$ muitipole does not appear to be a problem.

The following 2 sections apply the above approach to two correction coil designs for ISABELLE. The first is a seperate correction magnet which contains all the correction coils and there are no correction coils in the dipole and quadrupole. In the second, the correction coils remain in the dipole and quadrupole but are now of the multi-layer universal type described above. 


\section{LAYER UNIVERSAL CORRECTION MAGNET}

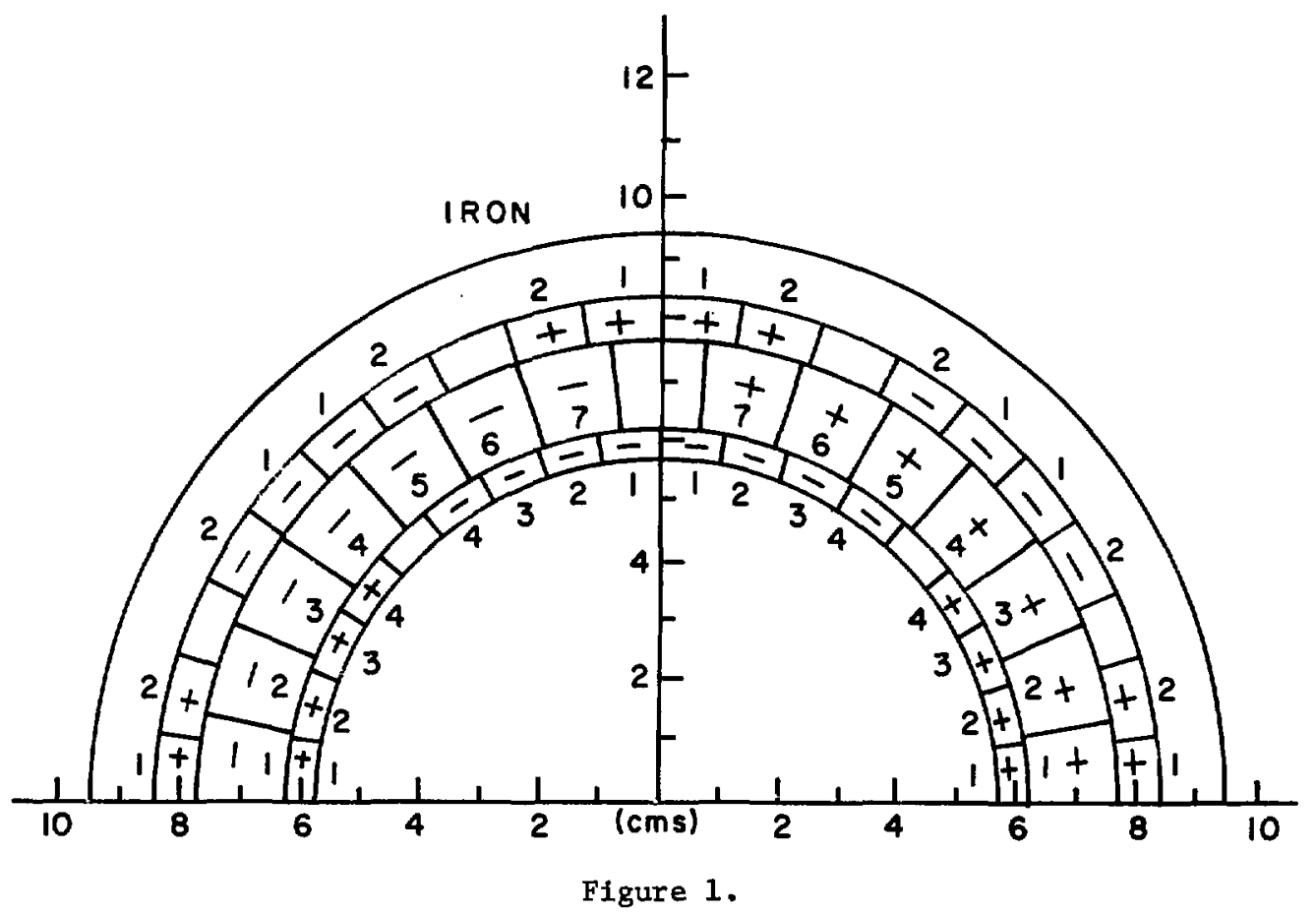


A table of the layers of a universal correction magnet. $n$ is the series of integers $n=0,1,2,3 \ldots$ The repeating interval is the interval in $\theta$ after which the winding pattern repeats itself. The location of the return current is for current blocks in the first interval. The multipole field is given by $B_{o} b_{m} x^{m}$, where $B_{o}$ is some relevant standard field.

\begin{tabular}{|c|c|c|c|c|}
\hline Layer & $\begin{array}{l}\text { Field } \\
\text { Multipoles }\end{array}$ & $\begin{array}{l}\text { Current } \\
\text { Harmonics, } k\end{array}$ & $\begin{array}{l}\text { Repeating } \\
\text { Interval }\end{array}$ & $\begin{array}{l}\text { Location of } \\
\text { Return Current }\end{array}$ \\
\hline 1 & $b_{0}, b_{2}, b_{4}, \ldots$ & $(2 n+1) \times 1$ & $\pi$ & $\pi-\theta$ \\
\hline 2 & $b_{1}, b_{5}, b_{9}, \ldots$ & $(2 n+1) \times 2$ & $\pi / 2$ & $\pi / 2-\theta$ \\
\hline 3 & $b_{3}, b_{11}, b_{19}, \cdots$ & $(2 n+1) \times 4$ & $\pi / 4$ & $\pi / 4-\theta$ \\
\hline 4 & $b_{7}, b_{23}, \ldots$ & $(2 n+1) \times 8$ & $\pi / 8$ & $\pi / 8-\theta$ \\
\hline 5 & $b_{15}, b_{47}, \cdots$ & $(2 n+1) \times 16$ & $\pi / 16$ & $\pi / 16-\theta$ \\
\hline- & $\cdots$ & -- & --- & $-\infty$ \\
\hline Layer $s$ & $b_{k-1}$ & $(2 n+1) \times 2^{s-1}$ & $\pi / 2^{s-1}$ & $\pi / 2^{s-1}-\theta$ \\
\hline
\end{tabular}

II. A SEPARATE CORRECTION MAGNET FOR ISABELLE

In this design, all the correction coils are placed in a magnet which is $1.6 \mathrm{~m}$ long and which is near each of the normal cell quadrupoles. The geometry of this design is shown in Fig. 1 .

Table II lists the required strengths for the correction field multipoles $b_{1}, b_{2}, b_{3}, b_{4}$, and $b_{5}$ for the ISABELLE accelerator when all the correction coils are located in a seperate 1.6 meter long magnet near the quadrupoles.

TABLE II.

The strengths of the correction field multipoles required in ISABELLE, when the correction coils are located in a separate 1.6. meter long magnet near the quadrupoles. The multipole field is given by $B_{o} b_{m} x^{m}$, where $B_{o}$ is the main dipole field. The quantities $b_{m} R^{m}$ give the multipole field relative to $B_{0}$ at $R=7.3 \mathrm{~cm}$.

\begin{tabular}{lll}
$\mathrm{m}$ & \multicolumn{1}{c}{$\begin{array}{c}\mathrm{b}_{\mathrm{m}} \\
\left(\mathrm{cm}^{-m}\right)\end{array}$} & $b_{\mathrm{m}} R^{\mathrm{m}}$ \\
\hline 1 & $3.0 \mathrm{E}-3$ & 0.022 \\
2 & $26.4 \mathrm{E}-4$ & 0.140 \\
3 & $26.4 \mathrm{E}-5$ & 0.106 \\
4 & $40.0 \mathrm{E}-6$ & 0.110 \\
5 & $1.4 \mathrm{E}-6$ & 0.031
\end{tabular}


This design has 3 layers corresponding to the first 3 layers described in the first section. Each layer has a maximum current density of $\mathrm{S}_{\max }=10 \mathrm{kA} / \mathrm{cm}^{2}$ to obtain the required field multipole corrections. The arrangement of the layers, which one is the inner or middle or outsr layer, is chosen to minimize the radial width of the 3 1ayers.

The middle layer has 7 independently excited coils and can generate the field multipole $b_{0}, b_{2}, b_{4}, b_{6}, b_{8}, b_{10}, b_{12}$.

The inner layer has 4 independent coils and can generate the field multipole $b_{1}, b_{5}, b_{9}, b_{13}$. The outer layer has 2 independent coils and can generate the field multipole $b_{3}, b_{11}$. All together there are 13 independent excited coils that can generate all the multipoles from $b_{0}$ to $b_{13}$ with the exception of $b_{7}$.

Higher field multipoles past $b_{13}$ can be controlled by adding more independently excited coils. However, as long as one is restricted to 3 layers certain multipoles can not be generated. These are the multipoles $b_{7}, b_{15}, b_{23}, b_{31} \ldots$ A fourth layer is required to generate $b_{7}$ and $b_{23}$, and a fifth layer to generate $b_{15}$ and so on.

\section{UNIVERSAL CORRECTION COILS IN THE ISABELLE MAGNETS}

In this design, the correction coils are in the dipoles and quadrupoles of ISABELLE. There are sti11 3 layers. Two layers are in the dipole and one layer is in the quadrupole.

Table III lists the required strengths for correction field multipoles $b_{1}$ to $b_{5}$ in ISABELLE, when $b_{2}, b_{3}, b_{4}$ are located in the dipoles, and $b_{1}, b_{5}$ are located in the quadrupoles. An estimate of the expected values for the systematic multipoles $b_{6}$, $b_{8}, b_{10}, b_{12}$ in the dipoles are also given. The values given for $b_{m} R^{m}$, the multipole field relative to $B_{0}$ at $R=7.3 \mathrm{~cm}$ show that the systematic multipoles can be corrected near the injection field level without requiring additional correction capacity; at higher field levels, correcting the systematic multipoles is not possible without additional correction capacity.

In the dipoles, shown in Fig. 2, the outer layer has 7 independently, excited correction coils which can generate $b_{0}, b_{2}, b_{4}$, $b_{6}, b_{8}, b_{10}, b_{12}$. The inner layer has 2 independent coils which can generate $b_{3}$ and $b_{11}$. Each layer has a maximum current density of $8.5 \mathrm{kA} / \mathrm{cm}^{2}$ to obtain the required field multipole correction.

In the quadrupoles, shown in Fig. 3, the one layer has 4 independently excited correction coils wich can generate $b_{1}, b_{5}, b_{9}$ $b_{13}$. This layer has a required current density of $10.5 \mathrm{kA} / \mathrm{cm}^{2}$ to obtain the required field multipole corrections. The quadrupole also has another layer which is the dipole corrector for the closed orbit correction system which are not being considered here. 
TABLE III.

The required strengths for the field correction multipoles $b_{1}$ to $b_{5}$, when $b_{2}, b_{3}, b_{4}$ are located in the dipoles and $b_{1}, b_{5}$ are 10cated in the quadrupoles. An estimate of the expected values for the systematic multipoles $b_{6}, b_{8}, b_{10}, b_{12}$ in the dipoles, and $\mathrm{b}_{9}, \mathrm{~b}_{13}$ in the quadrupoles are al so given. The $\mathrm{b}_{\mathrm{m}} \mathrm{R}^{\mathrm{m}}$ give the multipole fields relative to $B_{0}$ at the radius $R=7.3 \mathrm{~cm}$.

\begin{tabular}{|c|c|c|c|}
\hline $\mathbf{m}$ & $\left(\mathrm{cm}^{-}\right.$ & & $b_{\mathfrak{m}} \mathrm{R}^{\mathbb{m}}$ \\
\hline 1 & 3.0 & $E-3$ & 0.022 \\
\hline 2 & 6.0 & $E-4$ & 0.032 \\
\hline 3 & 4.0 & $E-5$ & 0.016 \\
\hline 4 & 5.0 & $E-6$ & 0.014 \\
\hline 5 & 1.5 & $E-6$ & 0.031 \\
\hline 6 & 1.7 & $E-9$ & 0.00026 \\
\hline 8 & 7.6 & $E-10$ & 0.0061 \\
\hline 10 & -3.5 & E-11 & 0.015 \\
\hline 12 & -2.70 & $E-14$ & 0.00062 \\
\hline 9 & -3.0 & $E-10$ & 0.018 \\
\hline 13 & -3.2 & E-13 & 0.0544 \\
\hline
\end{tabular}

It appears that the multilayer unversal correction coil will often require lower current densities than those required by a correction design which has a sequence of nested layers, each layer generating a pure multipole. For example, one ISABELLE design has seperate $b_{2}$ and $b_{4}$ correction coils in two layers in the dipoles, and the required maximum current density in the sextupole layer is $S_{\max }=17 \mathrm{KA} / \mathrm{cm}^{2}$. In the above universal coil design, the $b_{2}$ and $b_{4}$ multipoles are generated in one layer and the required maximum current density is 8.5 $\mathrm{KA} / \mathrm{cm}^{2}$. The considerably lower current density found for the universal coil design probably results from more efficient use of the radial space occupied by the correction coils. In the nested pure multipole coil design, the $b_{4}$ layer occupies about the same space as the $b_{2}$ layer, although the required current density for the $b_{4}$ layer is much lower. 
9 COILS IN TWO LAYERS IN THE DIPOLE

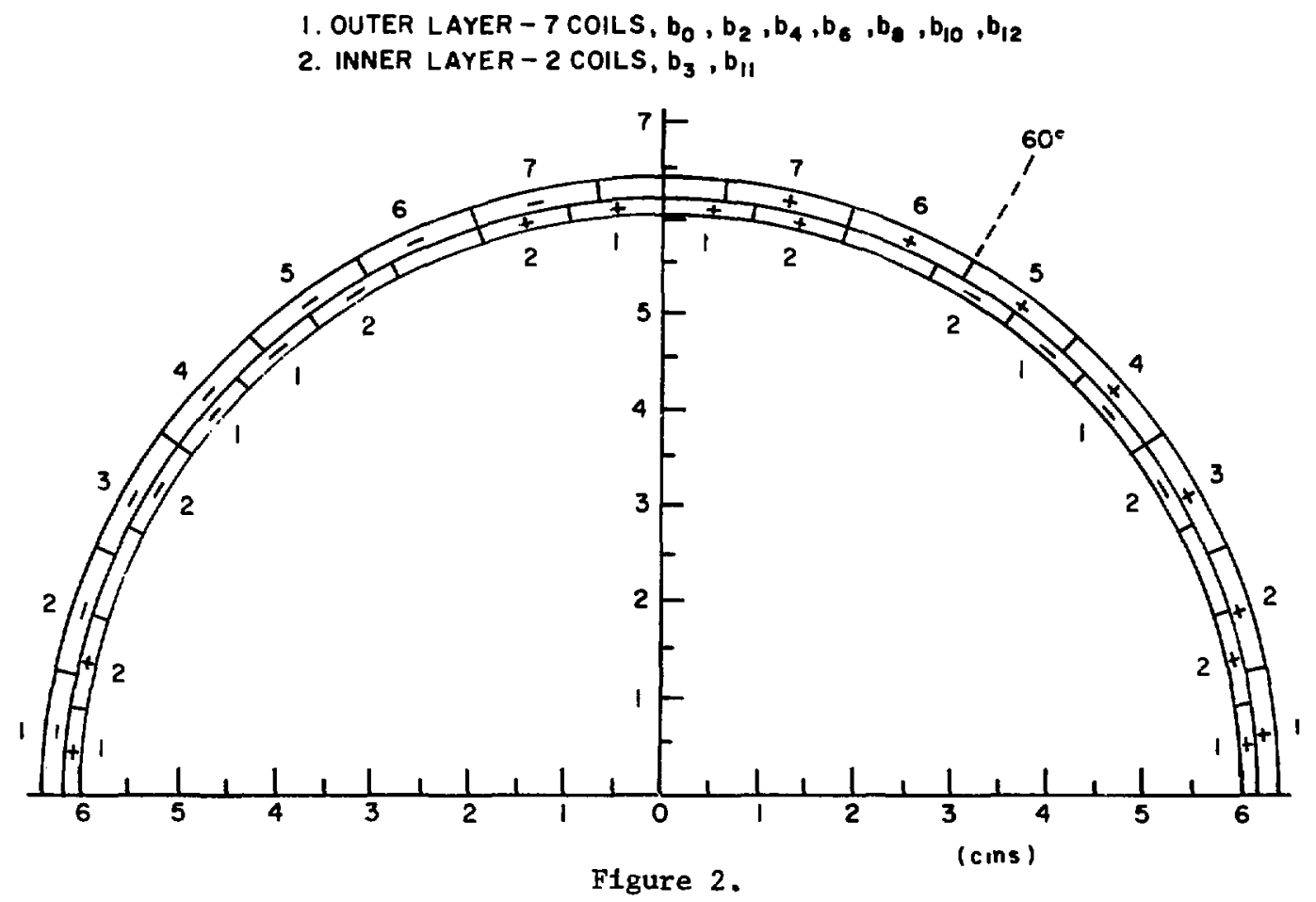




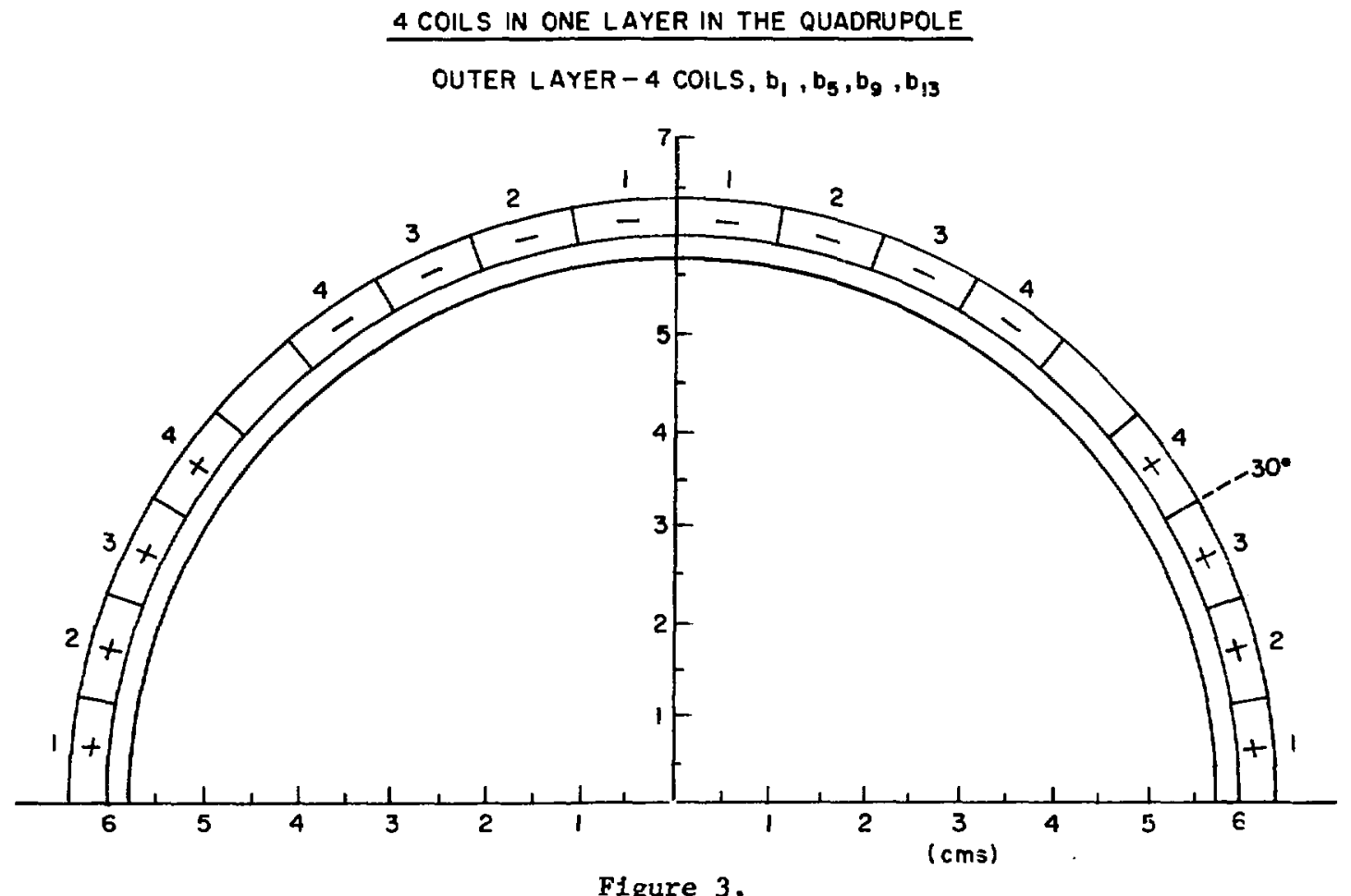

Figure 3. 